\title{
Comparative evaluation of silver nanoparticle gel and chlorhexidine gel as an adjunct to scaling and root planing in management of chronic periodontitis-A clinico-microbiological study
}

\author{
Udbhabana Kalita $^{1 *}$, Varun Kumar Tyagi ${ }^{2}$, Swyeta Jain Gupta $^{3}$, Shubhra Vaish ${ }^{4}$, Nikhil Sharma ${ }^{5}$ \\ ${ }^{1,2}$ Post Graduate Student, ${ }^{3}$ Reader, ${ }^{4}$ Professor, ${ }^{5} \mathrm{HOD},{ }^{1,2}$ Dept. of Periodontics and Oral Implantology, ${ }^{3-5}$ Dept. of Periodontology and \\ Implantology, ${ }^{1,2}$ ITS Dental College, Ghaziabad, Uttar Pradesh, ${ }^{3-5}$ IT.S-CDSR Muradnagar, Ghaziabad, Uttar Pradesh, India
}

*Corresponding Author: Udbhabana Kalita

Email: udbhabanakalita_mds19_22@its.edu.in

\begin{abstract}
Introduction: Bacteria play an important role in the development of periodontitis and chlorhexidine (CHX) is considered "gold standard" in its treatment. Silver ions being strong antiseptics have been used in dentistry for a long time. Therefore the aim of our study was to compare the efficacy of chlorhexidine gel and megaheal gel containing silver nanoparticles in treatment of patients suffering from chronic periodontitis.

Materials and Methods: 20 patients who had chronic periodontitis were selected who were divided randomly and equally into 2 groupsChlorhexidine gel group and Megaheal gel group. Subgingival plaque samples were taken from selected pocket before and after treatment and the number of colony forming units per millilitre was determined.

Results: The CFU count of anaerobic bacteria was found to be lower for Megaheal gel group as compared to Chlorhexidine gel group Conclusion: Thus, Megaheal gel was found to be more effective in reducing count of anaerobic bacteria than Chlorhexidine group.
\end{abstract}

Keywords: Silver nanoparticle gel, Chlorhexidine gel, Chronic periodontitis.

\section{Introduction}

The problems caused by periodontitis have long been identified as a serious health issue in many populations for a long time. ${ }^{1}$ Chronic periodontitis has a slow to moderate rate of disease progression, and is often associated with local predisposing factors: dental plaque, subgingival calculus deposits, some iatrogenic factors and systemic diseases, such as diabetes mellitus. ${ }^{2}$ The severity and extent of periodontal tissue damage varies from person to person and depends largely on immune responses of the individual to microorganisms. ${ }^{3}$

Chlorhexidine is an effective antimicrobial agent. It is a cationic bisbiguanide with broad antibacterial activity, ${ }^{4}$ low mammalian toxicity and possesses strong affinity for binding to skin and mucous membranes. ${ }^{5}$ Assessment of a gel formulation as an alternative means of delivering chlorhexidine to the teeth and oral cavity has proven problematic as much depends on the ability of the patient to deliver the gel to the appropriate areas of the mouth. Chlorhexidine gel does not penetrate easily to areas away from its site of application; ${ }^{6}$ thus, any assessment of the effects of the gel depends on the amount of gel reaching the appropriate area of the mouth at the appropriate period of time. $^{7}$

Also, silver is a non-toxic, powerful disinfectant, which can significantly reduce bacterial infection. ${ }^{8}$ The use of silver as an antiseptic is very frequent nowadays, but it has not been tested sufficiently in the field of periodontics. The effects of silver ions are mostly based on three mechanisms:

1. Interaction with DNA of bacteria

2. The destruction of the cell membrane

3. The blocking of essential enzymes that regulate transport of electrons. ${ }^{9}$
Silver has a long lasting bacteriostatic effect because it is bind to proteins tissue and chlorides.

\section{Materials and Methods \\ Patient selection criteria}

The presented clinical study was performed at the Department of Periodontology and Oral Implantology, I.T.S Centre for Dental Studies \& Research, Ghaziabad, India. The research protocol was reviewed and approved by the Ethical Committee of the Institution. All patients were educated about the study and then written consent was taken before enrolment in the study. The study was conducted for a period of three months. A flow diagram for complete methodology is presented in Fig. 1.

Twenty patients of both the sexes participated in the study and were divided into two groups. The first group received Chlorhexidine gel and the second group received megaheal gel after scaling and root planing.

The inclusion criteria of the study was

1. Patients in age range: $18-65$ years

2. Patients who were systemically healthy

3. Patients with diagnosis of chronic periodontitis

4. Patients having at least 20 teeth present excluding third molars.

5. Patients with presence of at least 2 teeth, with probing depth $\geq 5 \mathrm{~mm}$.

6. Clinical attachment loss of $\geq 5 \mathrm{~mm}$, in 2 quadrants.

\section{The study excluded}

1. The patients whose treatment of periodontitis was conducted more than 12 months ago

2. The presence of systemic diseases that may affect the treatment of periodontal disease 
3. The use of antibiotics and anti-inflammatory drugs in the last 6 months,

4. Pregnancy, lactation and the use of contraception drugs as well as systemic infections of the oral cavity.
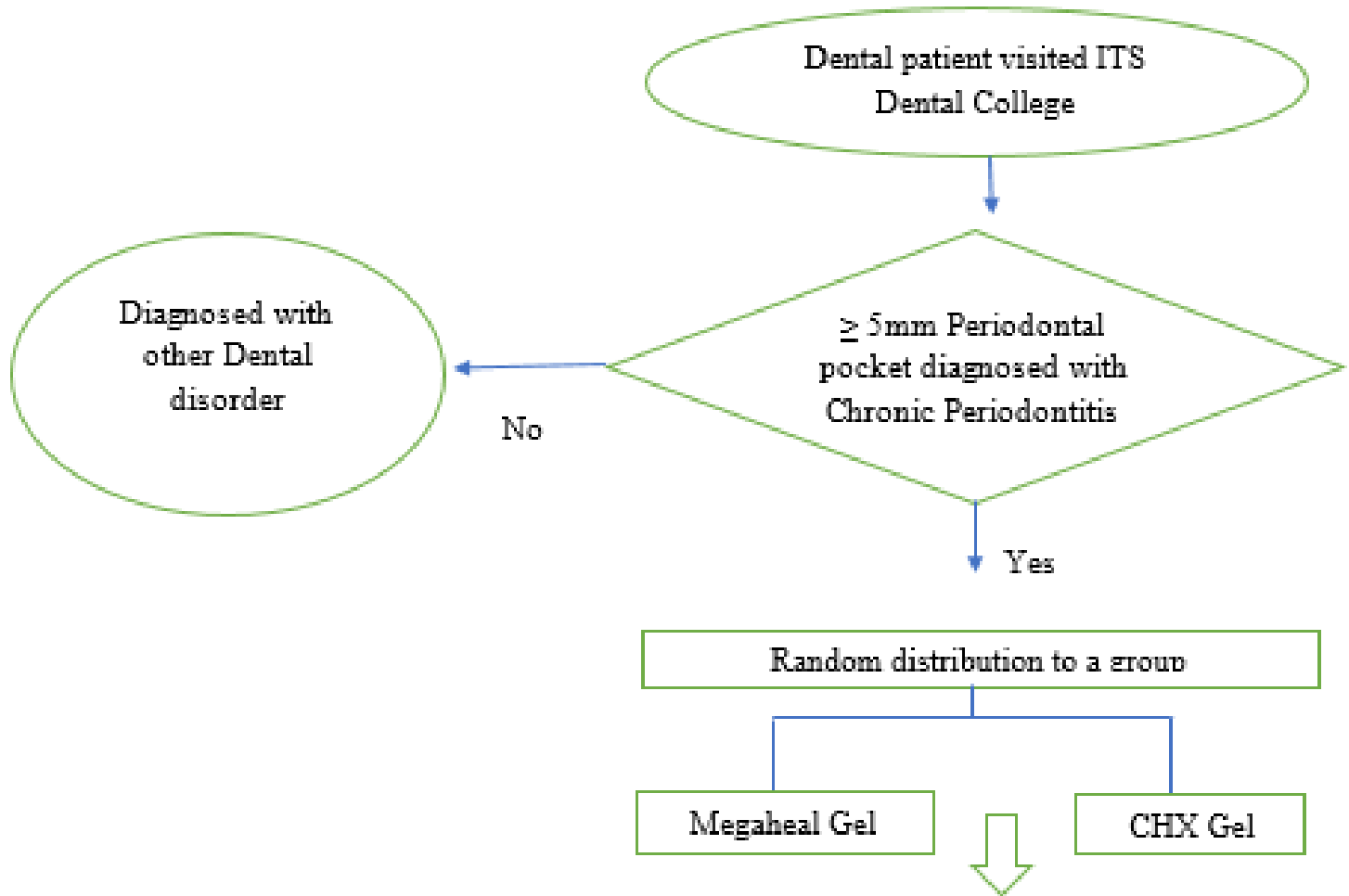

No of colony forming units per millilitre is determined in the 2 groups

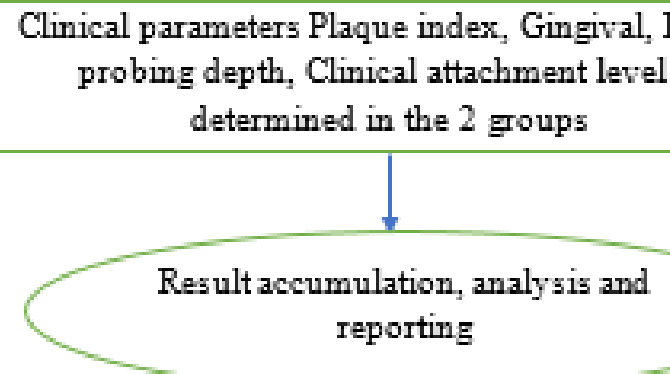

Fig. 1: Study design

\section{Patient's allocation and procedure}

Patients were assigned equally and randomly to one of the two groups $(\mathrm{N}=20)$. Envelopes containing identification for treatment groups were enclosed, mixed, and then numbered. Each participant was randomly allocated to one of the following group.
All teeth were measured (excluding third molars) with a CPITN probe. For the assessment of oral hygiene the following indices were used:

1. Plaque index $(\mathrm{PI})^{10}$, measured on four sides (mesiovestibular, vestibular, disto-vestibular, oral) and score marked with values $0-3$.

2. Gingival index $(\mathrm{GI})^{10}$, measured on the same four sides and score marked with $0-3$. The GI score of 2 and 3 indicated bleeding on probing (BOP). 
3. Periodontal pocket depth (PPD) ${ }^{11}$ in $\mathrm{mm}$ - the distance from the free gingiva margin to the bottom of the periodontal pocket.

4. In order to assess the periodontal status, the clinical attachment level $(\mathrm{CAL})^{11}$ - the distance from the cemento-enamel junction to the bottom of the periodontal pocket, was measured.

5. Also plaque sample was taken from the periodontal pocket at baseline and after 3 months of application of gel. Microbiological analysis was carried out for the samples collected. The sample was scraped by a Gracey curette from the root surface and placed in phosphate buffer saline $(5 \mathrm{ml})$.Automatic pipette was used during the dilution procedure. The dilution procedure was carried out at -1 and -2.Trypticase soy agar plates were prepared. A bacteriological loop was used to spread the PBS over the plates. The plates were then kept incubated for 48 hours. Anaerobic colonies formed were counted on a colony counter.

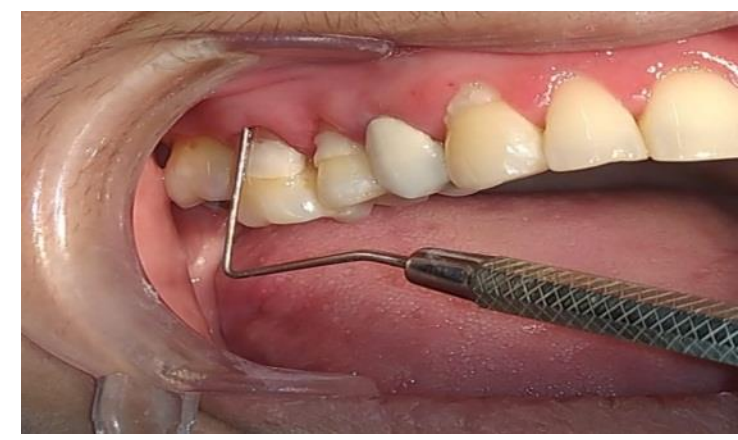

Fig. 2: Preoperative pic (CHX group)

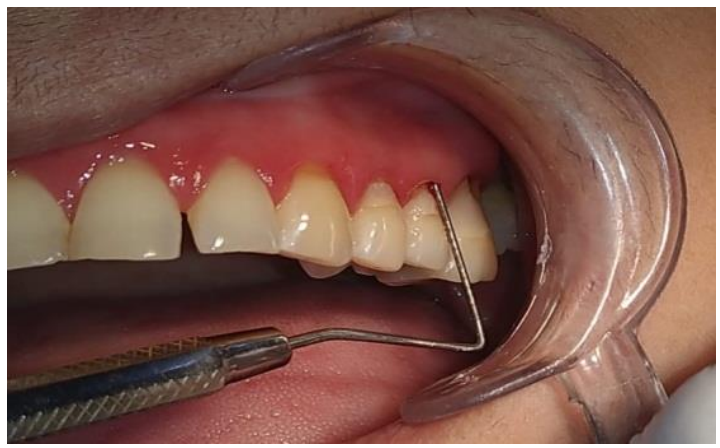

Fig. 3: Pre- operative pic (Megaheal group)

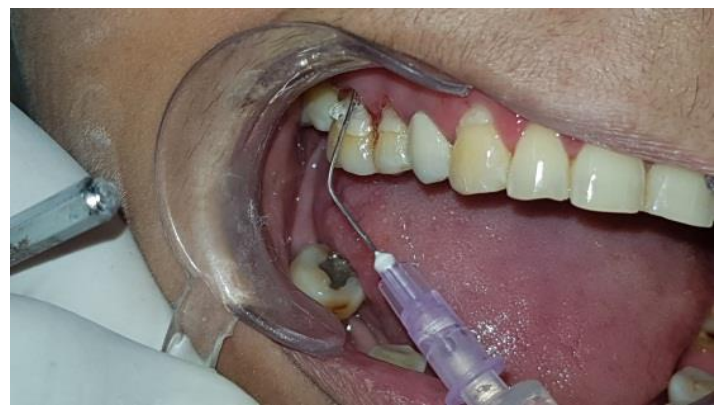

Fig. 4: Application of CHX

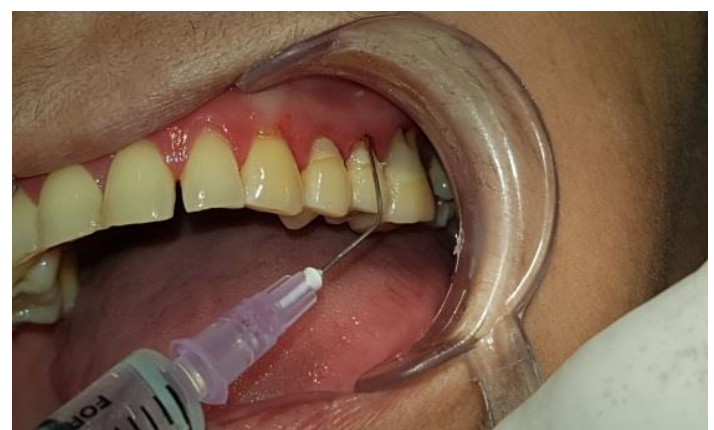

Fig. 5: Application of Megaheal

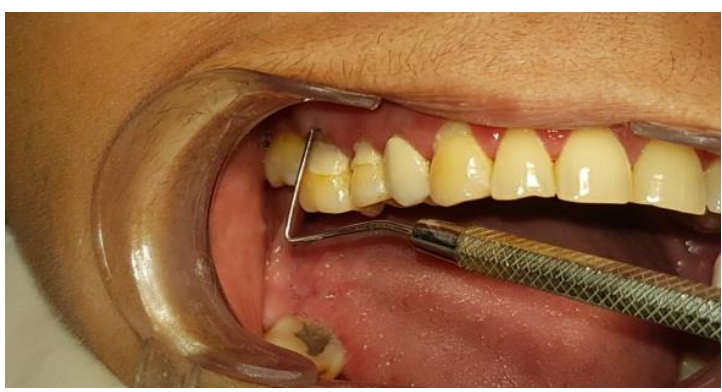

Fig. 6: Post operative pic (CHX group)

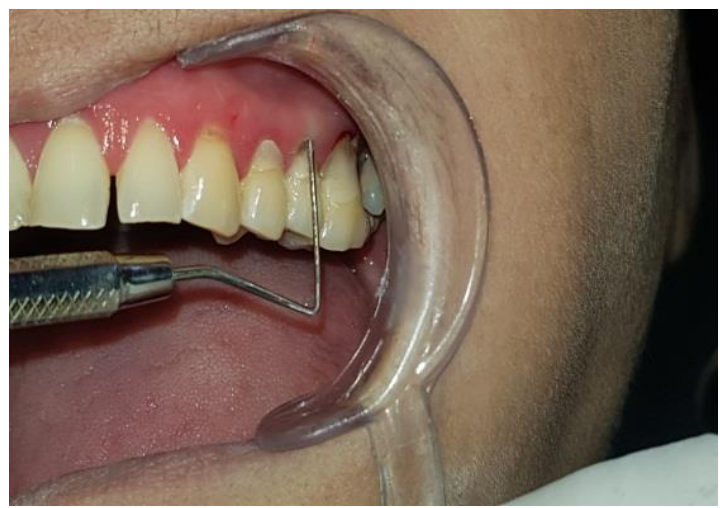

Fig. 7: Post operative pic (Megaheal group)

Complete statistical analysis of data was done with the help of statistical software package, SPSS Statistics 18. Variables were presented as mean value \pm standard deviation (SD).One way ANOVA test was used for evaluation of distribution of clinical data. A statistical significance within and between groups was tested by t-test for the paired and independent samples. All the analyses were estimated at $\mathrm{p}<$ 0.001 level of statistical significance. 


\section{Results}

The reduction in Plaque Index, Gingival Index, Pocket Probing Depth and Clinical Attachment level was found to be significant from baseline to 3 months between the groups. The microbiological analysis showed a higher reduction in CFU (Colony Forming Units) in Group B as compared to Group A from baseline to 3 months. (Graph 1 -3, Table 1)

Table 1: Mean values of clinical parameters Plaque index (PI), Gingival index (GI), Pocket probing depth (PPD) and Clinical Attachment level (CAL) at baseline and 3 months after treatment

\begin{tabular}{|l|c|c|c|c|}
\hline $\begin{array}{c}\text { Groups } \\
\text { (before/after } \\
\text { treatment) }\end{array}$ & $\begin{array}{c}\text { PI } \\
\text { mean } \pm \text { SD }\end{array}$ & $\begin{array}{c}\text { GI } \\
\text { mean } \pm \text { SD }\end{array}$ & $\begin{array}{c}\text { PPD } \\
\text { mean } \pm \text { SD }\end{array}$ & $\begin{array}{c}\text { CAL } \\
\text { mean } \pm \text { SD }\end{array}$ \\
\hline \multicolumn{5}{|c|}{ CHX $(\mathrm{n}=10$} \\
\hline Baseline & $0.342 \pm 0.142$ & $1.36 \pm 0.121$ & $5.7 \pm 0.483$ & $6.7 \pm 0.483$ \\
\hline 3 months & $0.327 \pm 0.139$ & $1.343 \pm 0.117$ & $4.4 \pm 0.516$ & $5.2 \pm 0.421$ \\
\hline P1 & $<0.001$ & $<0.001$ & $<0.001$ & $<0.009$ \\
\hline Megaheal (n=10) & $0.299 \pm 0.130$ & $1.37 \pm 0.102$ & $5.7 \pm 0.823$ & $6.7 \pm 0.823$ \\
\hline Baseline & $0.284 \pm 0.132$ & $1.35 \pm 0.104$ & $4.4 \pm 0.516$ & $5.5 \pm 0.527$ \\
\hline 3 Months & $<0.001$ & $<0.001$ & $<0.001$ & $<0.001$ \\
\hline P2 & \multicolumn{5}{|l}{} \\
\hline
\end{tabular}

Graph 1: Plaque index (PI) and Gingival index(GI) at baseline and re-evaluation after 3 months in both groups.

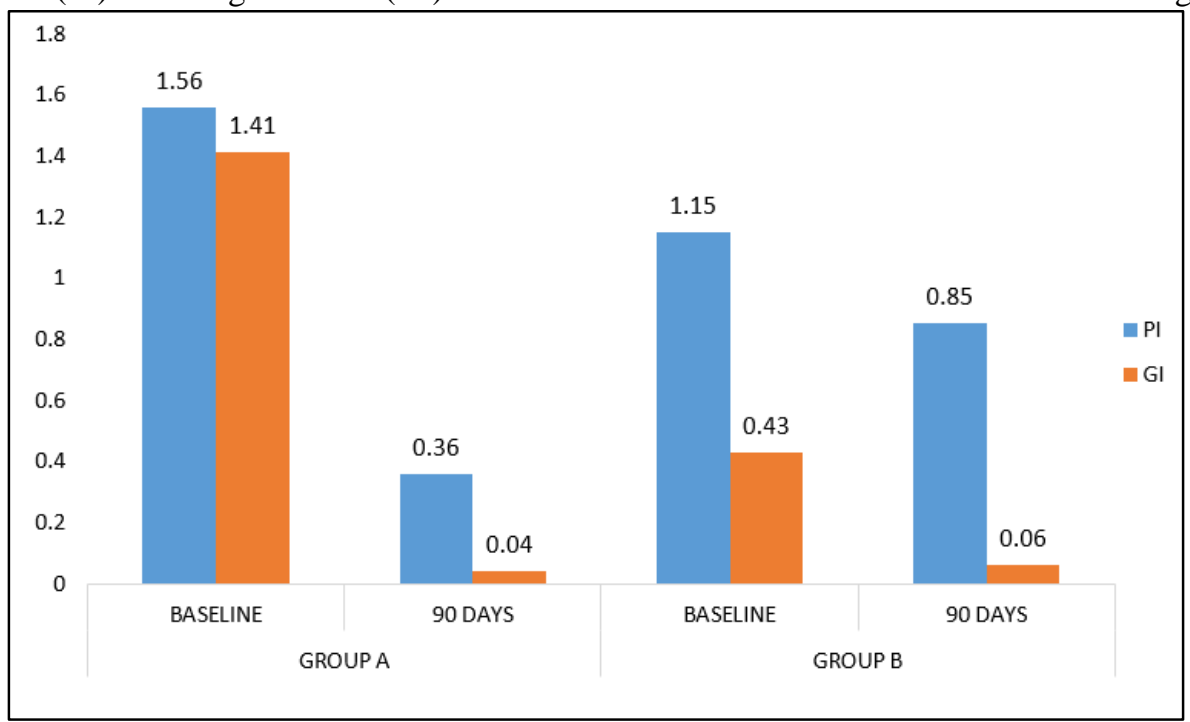

Graph 2: Pocket probing depth (PPD) and Clinical Attachment level (CAL) at baseline and re-evaluation after 3 months in both the groups

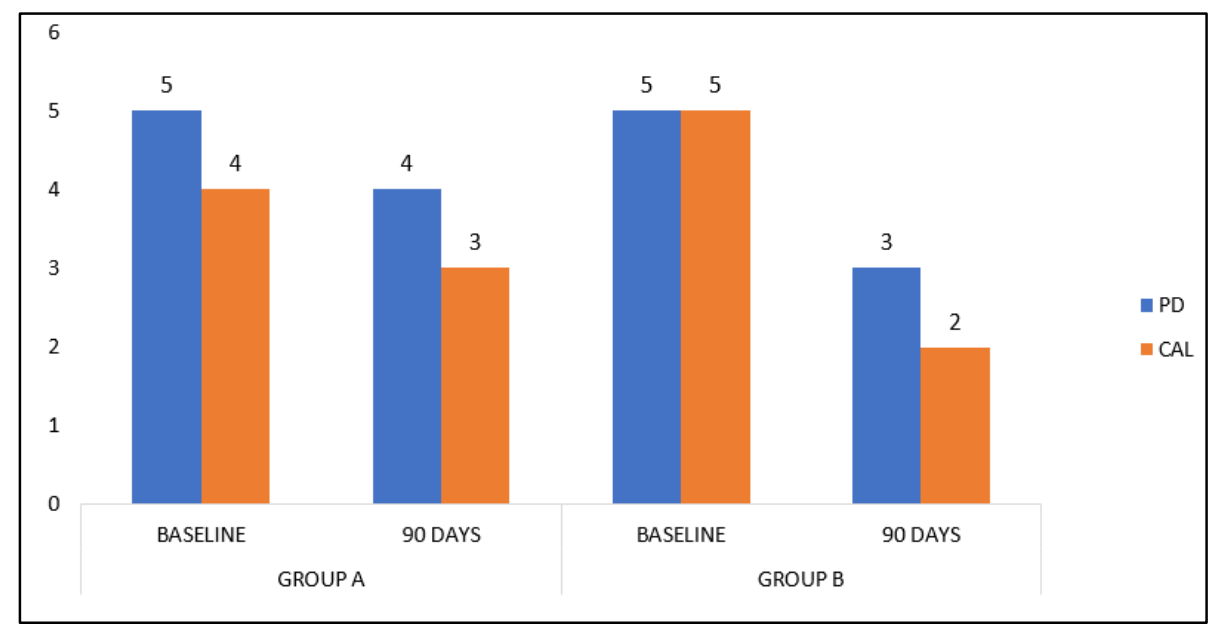


Graph 3: Colony Forming Units (CFUs) at baseline and re-evaluation after 3 months

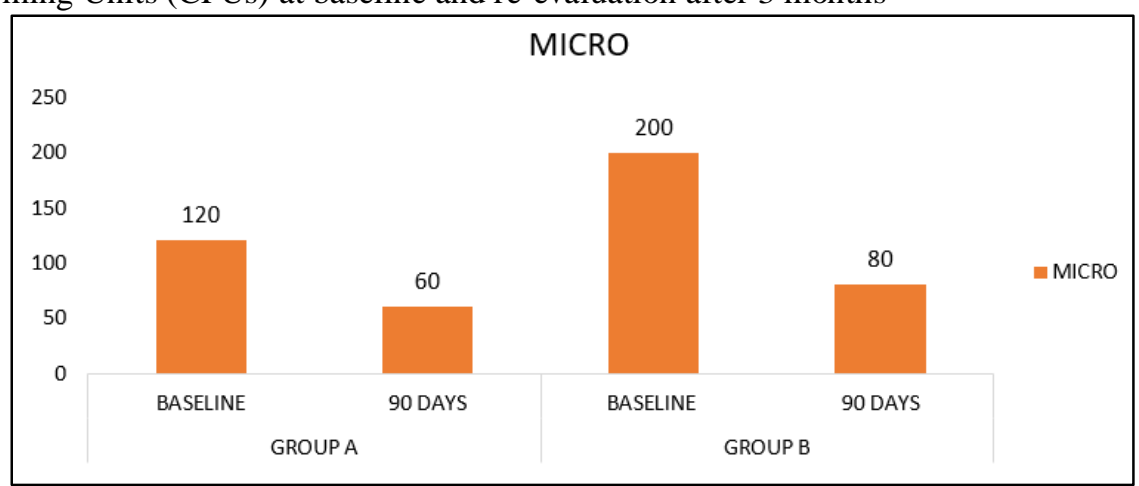

\section{Discussion}

Our results showed that the CHX and megaheal have an equal, statistically significant effect on all studied clinical parameters PI, GI, PPD, CAL in the patients with a chronic form of periodontal disease. In the study, we demonstrated that megaheal gel led to a reduction in PPD significantly because of its successful antibacterial efficacy. This efficacy against periodontal pathogenic species was also shown in other studies. ${ }^{12}$ The compounds that contain silver are attractive because of their strong antimicrobial activity, high stability and wide-spread antibacterial spectrum. ${ }^{13}$ For the assessment of oral hygiene, PI was used, which was statistically significantly reduced after the treatment in both of the tested groups $(p=0.001)$. We can explain these results by the fact that silver ions prevent adhesion of bacteria and thus influence the prevention of biofilm formation which precedes creation of plaque on dental hard structure. Nowadays the emphasis on the testing of silver activity against periodontal pathogens from the biofilm has been increased recently. The study by Lu et al. also focused on the antibacterial effect of silver nano-particles of various sizes on anaerobic bacteria, the results of the study showed that the smallest tested nano-particles $(5 \mathrm{~nm})$ showed greater antimicrobial effects compared to larger particles (15-55 $\mathrm{nm}){ }^{14}$ There are positive antibacterial effects of different sizes of silver nanoparticles on anaerobic bacteria; the assumption is that this effect stems from silver astringent potential associated with the direct or even indirect antibacterial effects of silverions. ${ }^{15}$ Passing through the bacterial cell wall, silver ions prevent the replication of the DNA molecule. In this way, the interactive inactivation of bacterial proteins occurs and as a result of the catalytic activity of silver, oxygen radicals are released, which cause structural damage inside the bacteria. ${ }^{16}$ This phenomenon leads to damage or even the death of bacteria.

\section{Conclusion}

The use of adjuvant antiseptics within the SRP for patients with chronic forms of periodontal disease has been justified. In our study, the tests showed that CHX gel and megaheal gel as adjuvant antiseptics and as part of SRP, have practically the same statistically significant effect on all studied clinical parameters. In the Megaheal group, 3 months after therapy, a significant reduction of GI and PI was obtained compared to CHX group. This is of great importance, because megaheal was not used as the adjuvant antiseptic in this category of patients and, as the CHX was accepted as a "gold standard" of this type, it seems that silver ions should be re-evaluated for adjuvant treatment within the causal therapy of patients with chronic periodontal disease.

\section{Source of funding}

None.

\section{Conflict of interest}

None.

\section{References}

1. Lui J, Corbet EF, Jin L. Combined photodynamic and lowlevel laser therapies as an adjunct to nonsurgical treatment of chronic periodontitis. J Periodontal Res 2011;46(1):89-96.

2. Anitha V, Rajesh P, Shanmugam M, Priya BM, Prabhu S, Shivakumar V. Comparative evaluation of natural curcumin and synthetic chlorhexidine in the management of chronic periodontitis as a local drug delivery: a clinical and microbiological study. Indian J Dent Res 2015;26(1):53-56.

3. Pattnaik S, Anand N, Chandrasekaran SC, Chandrashekar L, Mahalakshmi K, Satpathy A. Clinical and antimicrobial efficacy of a controlled-release device containing chlorhexidine in the treatment of chronic periodontitis. Eur J Clin Microbiol Infect Dis 2015;34(10):2103-10.

4. Genovesi A, Barone A, Toti P, Covani U. The efficacy of $0.12 \%$ chlorhexidine versus $0.12 \%$ chlorhexidine plus hyaluronic acid mouthwash on healing of submerged single implant insertion areas: a short-term randomized controlled clinical trial. Int J Dent Hyg 2017;15(1):65-72.

5. Denton GW. Chlorhexidine: a WHO essential drug. Lancet. 1984;2(8401):517.

6. Mohammad Samiei, Klara Janjić, Barbara Cvikl, Andreas Moritz and Hermann Agis, The role of sclerostin and dickkopf1 in oral tissues - A review from the perspective of the dental disciplines, J Periodontol 1967;38(6):Suppl:610-6.

7. Saxén L, Niemi ML, Ainamo J. Intraoral spread of the antimicrobial effect of a chlorhexidine gel. Scand J Dent Res 1976;84(5):304-7.

8. Usher PJ. Oral hygiene in mentally handicapped children. A pilot study of the use of chlorhexidine gel. Br Dent J 1975;138(6):217.

9. Qing Y, Cheng L, Li R, Liu G, Hang Z, Wang J et al. Potential antibacterial mechanism of silver nanoparticles and the 
optimization of orthopedic implants by advanced modification technologies. Int J Nanomedicine 2018;13:3311.

10. Löe H. The Gingival Index, the Plaque Index and the Retention Index Systems. J Periodontol 1967;38(6):Suppl:610-6.

11. Choi YM, Lee JY, Choi J, Joo JY. Effect of root planing on the reduction of probing depth and the gain of clinical attachment depending on the mode of interproximal bone resorption. $J$ Periodontal Implant Sci 2015;45(5):184-9.

12. Tran P L, Luth K, Wang J, Ray C, Mehta D, Moeller KW et al. Efficacy of a silver colloidal gel against selected oral bacteria in vitro. F1000Res 2019;8:267.

13. McCann M, Curran R, Ben-Shoshan M, Mckee V, Tahir AA, Devereux M, et al. Silver (I) complexes of 9anthracenecarboxylic acid and imidazoles: Synthesis, structure and antimicrobial activity. Dalton Trans 2012;41(21):6516-27.

14. Abbasi E, Milani M, Fekri Aval S, Nasrabadi HT, Nikasa P, Joo SW et al. Silver nanoparticles: Synthesis methods, bio- applications and properties. Crit Rev Microbiol 2016;42(2):173-80

15. Burduşel AC, Gherasim O, Grumezescu AM, Mogoantă L, Ficai A, Andronescu E. Biomedical Applications of Silver Nanoparticles: An Up-to-Date Overview. Nanomaterials (Basel) 2018;8(9):681.

16. Dakal TC, Kumar A, Majumdar RS, Yadav V. Mechanistic Basis of Antimicrobial Actions of Silver Nanoparticles. Front Microbiol 2016;7:1831.

How to cite the article: Kalita U, Tyagi VK, Gupta SJ, Vaish S, Sharma N. Comparative evaluation of silver nanoparticle gel and chlorhexidine gel as an adjunct to scaling and root planing in management of chronic periodontitis-A clinico-microbiological study. $J$ Dent Specialities 2019;7(2):89-94. 